\title{
Clinical importance of puerarin for nursing of patients with glaucoma.
}

\author{
Linna Hao ${ }^{1}$, Dezhi $\mathrm{Lu}^{2}$, Xiaoxu Zhao ${ }^{3}$, Xinying Liu ${ }^{1 *}$ \\ ${ }^{1}$ Department of 2nd Ophthalmology, the First Branch of Mudanjiang Medical University Affiliated Hongqi Hospital, \\ Mudanjiang, Heilongjiang, P.R. China
}

${ }^{2}$ Department of Pediatric Surgery, Mudanjiang Medical University Affiliated Hongqi Hospital, Mudanjiang, Heilongjiang, P.R. China

${ }^{3}$ Department of 1st Ophthalmology, the First Branch of Mudanjiang Medical University Affiliated Hongqi Hospital, Mudanjiang, Heilongjiang, P.R. China

\begin{abstract}
To determine whether clinical nursing of Puerarin can improve the visual outcome for patients with primary open angle glaucoma. A total of 108 patients with primary open angle glaucoma were recruited and received treatment with Puerarin or placebo in eye drop instillation using a 5-mL plastic bottle of artificial tear solution. The visual field defect and clinical characteristics intraocular pressure, graded corneal erosion, Tear Break-Up Time (TBUT), and subjective discomfort were analyzed after treatment with Puerarin. Outcomes showed that Puerarin treatment decreased intraocular pressure and inflammation score compared to placebo group. Puerarin treatment improved graded corneal erosion, TBUT, subjective discomfort and visual acuity, glaucoma visual field defect compared to placebo group. There was no safety issues were observed in either group. Ocular hyperemia was the most common treatment-related adverse event. In conclusion, these outcomes indicate that patients receiving Puerarin maintained better treatment adherence compared with control through 6 months of ontherapy evaluation, which suggest that Puerarin may provide improved long-term therapeutic outcomes for glaucoma patients.
\end{abstract}

Keywords: Glaucoma, Puerarin, Visual acuity.

Accepted on January 30, 2018

\section{Introduction}

Glaucoma is one of the leading causes of irreversible visual loss accompanying high intraocular pressure, optic neuropathy and/or specific visual field deficits [1]. Clinical reports have found that various factors contribute to the formation and development of glaucoma [2,3]. The ultimate goal for glaucoma patients is to restore patients' visual function and quality of vision.

Puerarin is extracted from Radix puerariae. Puerarin has many therapeutic effects for neurological dysfunction and inflammation [4]. Study found that Puerarin eye drops provided principle basis for treating eye diseases with Puerarin by systemic administration [5]. Previous study also showed that Puerarin eye drops enhanced ocular bioavailability and patient compliance [6]. However, the therapeutic effects of Puerarin on glaucoma have not well understood for glaucoma patients.

In this study, we investigated the therapeutic effects of Puerarin in a total of 108 patients with primary open angle glaucoma.
We analyze therapeutic outcomes of glaucoma patients in 6month Puerarin treatment.

\section{Materials and Methods}

\section{Patients}

The study was approved by the Ethics Committee of the Tianjin Children's Hospital. A total of 108 (54 male/54 female) glaucoma patients and 110 (54 male/56 female) healthy volunteers were required to write informed consent for all participants. The mean age was $58.3 \pm 12.3$ and $58.2 \pm 12.5 \mathrm{y}$ old in glaucoma patients and healthy volunteers, respectively. Patients with diabetes mellitus were excluded from this study. All patients were received treatment with Puerarin $(2 \mathrm{mg} / \mathrm{kg}$, Thermo Fisher, CA, USA, $\mathrm{n}=54)$ or placebo $(2 \mathrm{mg} / \mathrm{kg}$, Thermo Fisher, CA, USA, n=54).

\section{Inflammation score}

Criteria for evaluation were determined by inflammation severity score (primary efficacy criteria) for SPPSA. Mean 
inflammation severity score were evaluated within $96 \mathrm{~h}$ after puerarin or placebo treatment as described previously [7].

\section{Outcomes measurement}

Intraocular pressure measurement was performed using the Goldmann applanation tonometer. Visual field assay was analyzed using the Humphrey VF Analyzer (Carl Zeiss Meditec Inc., Dublin, CA, USA) with the standard 10-2 test pattern. Visual acuity was measured using a standard Japanese decimal VA chart and the corrected VA was calculated using the decimal visual acuity scale. The location of VF defects of each participant with glaucoma was determined by comparing the values of total deviation (TD).

\section{Statistical analysis}

All data were presented as mean \pm standard error. All data were analyzed using SPSS 17.0 (SPSS Inc., Chicago, IL, USA). The Student's t-test was used for comparisons between groups. ${ }^{*} \mathrm{P}<0.05$ were considered statistical differences.

\section{Results}

\section{Characteristics of glaucoma patients}

A total of 108 patients with primary open angle glaucoma were enrolled. There were 54 male and 54 female patients. There were 58 (male/female: 28/30) received Puerarin and 50 (male/ female: $26 / 24$ ) received placebo. The male to female ratio was not significantly different between the Puerarin and placebo group. The demographic and clinical characteristics of each group were shown in Table 1.

Table 1. Characteristics of glaucoma patients.

\begin{tabular}{lll}
\hline & Glaucoma patients & Healthy volunteers \\
\hline Male & 54 & 52 \\
\hline Female & 54 & 56 \\
\hline Age (y old) & $58.3 \pm 12.3$ & $58.2 \pm 12.5$ \\
\hline Puerarin & 58 & 0 \\
\hline Placebo & 50 & 0 \\
\hline
\end{tabular}

\section{Therapeutic effects of Puerarin for glaucoma patients}

The therapeutic outcomes between Puerarin and placebo group were analyzed. Outcomes demonstrated that Puerarin treatment decreased intraocular pressure and inflammation score compared to placebo. 6-month Puerarin treatment significantly improved graded corneal erosion, TBUT, subjective discomfort and visual acuity, glaucoma visual field defect compared to placebo group (Table 2).

Table 2. Therapeutic outcomes of Puerarin for glaucoma patients.

\begin{tabular}{|c|c|c|c|}
\hline Parameter & Placebo & Puerarin & $P$ value \\
\hline
\end{tabular}

\begin{tabular}{llll}
\hline Intraocular pressure $(\mathrm{mmHg})$ & $17.30 \pm 3.28$ & $15.45 \pm 2.86$ & 0.042 \\
\hline Inflammation score & $6.20 \pm 1.6$ & $3.5 \pm 2.0$ & 0.028 \\
\hline Corneal erosion (points) & $0.34 \pm 0.10$ & $0.15 \pm 0.08$ & 0.048 \\
\hline TBUT (s) & $5.94 \pm 2.39$ & $3.45 \pm 2.07$ & 0.027 \\
\hline Subjective discomfort (points) & $1.28 \pm 0.66$ & $0.90 \pm 0.40$ & 0.036 \\
\hline Visual acuity (LogMAR) & $0.328 \pm 0.104$ & $0.102 \pm 0.062$ & 0.031 \\
\hline Visual field defect (\%) & $62.10 \pm 16.20$ & $42.42 \pm 12.40$ & 0.026 \\
\hline
\end{tabular}

\section{Side effects of Puerarin for glaucoma patients}

The safety of Puerarin was analyzed for glaucoma patients. Side effects including headache, naupathia, vomiting, hypertension and stomach were not found for patients both Puerarin and placebo group. Ocular hyperemia was the most common treatment-related adverse event. The concentration of Puerarin was analyzed in urine and plasma samples. We observed urinary concentrations of Puerarin were $5.2 \pm 1.1$ $\mathrm{ng} / \mathrm{ml}$ and plasma levels of Puerarin were $42.3 \pm 18.4 \mathrm{ng} / \mathrm{ml}$ during the Puerarin treatment period (Table 3). These outcomes suggest that no safety problem were observed in either group.

Table 3. Side effects of Puerarin for glaucoma patients (n).

\begin{tabular}{lll}
\hline & Placebo & Puerarin \\
\hline Headache & 2 & 4 \\
\hline Naupathia & 1 & 2 \\
\hline Vomiting & 2 & 3 \\
\hline Hypertension & 3 & 4 \\
\hline Stomach & 3 & 4 \\
\hline Ocular hyperemia & 2 & 7 \\
\hline Urinary concentration $(\mathrm{ng} / \mathrm{ml})$ & 0 & $5.2 \pm 1.1$ \\
\hline Plasma level $(\mathrm{ng} / \mathrm{ml})$ & 0 & $42.3 \pm 18.4$
\end{tabular}

\section{Discussion}

This study investigated the therapeutic effects of clinical Puerarin nursing on a total of 108 patients with primary open angle glaucoma. We reported that Puerarin treatment significantly improved the intraocular pressure and inflammation score compared to placebo. We observed that Puerarin treatment markedly improved visual acuity and subjective discomfort. Findings in the current study indicate that Puerarin is safe for glaucoma patients and only ocular hyperemia was observed in this study.

Currently, Puerarin is reported to association with the attenuation of inflammation in arthritis [8]. Anti-inflammatory potency of Puerarin was also reported in lipopolysaccharideinduced inflammation [9]. In this study, we found that inflammation score was decreased by Puerarin treatment in patients with glaucoma. The inflammation and associated pain involved in dressing changes of burn patients were relieved 
by Puerarin treatment [10]. We reported that Puerarin decreased intraocular pressure and improved graded corneal erosion, TBUT, subjective discomfort and visual acuity, glaucoma visual field defect compared to placebo group during 6-month treatment, which suggest that Puerarin may a potential agent for the treatment of glaucoma.

A clinical efficacy and safety of Puerarin injection has been identified for coronary heart disease patients [11]. Efficacy and safety of Puerarin injection in treatment of diabetic peripheral neuropathy was also confirmed in randomized controlled trials [12]. Notably, no serious adverse Puerarin drug reactions were reported in the treatment of acute ischemic stroke. In this study, we reported that ocular hyperemia was the most common treatment-related adverse event. No other side effects were observed during the treatment.

In conclusion, findings in this study indicate that Puerarin reduced intraocular pressure and inflammation, improved visual acuity and subjective discomfort for glaucoma patients. These outcomes suggest that Puerarin eye drops may be regarded as a potential agent for the treatment of glaucoma patients.

\section{References}

1. Zhang Z, Yu M, Wang F, Dai Y, Wu Z. Choroidal thickness and open-angle glaucoma: a meta-analysis and systematic review. J Glaucoma 2016; 25:446-454.

2. Rodrigues GB, Abe RY, Zangalli C. Neovascular glaucoma: a review. Int J Retina Vitreous 2016; 2: 26.

3. Razeghinejad MRM, Masoumpour MM, Eghbal MHM, Myers JSM, Moster MRM. Glaucoma surgery in pregnancy: A case series and literature review. Iran J Med Sci 2016; 41: 437-445.

4. Wei SY, Chen Y, Xu XY. Progress on the pharmacological research of puerarin: a review. Chin J Natl Med 2014; 12: 407-414.

5. Deng X, Zhang Q, Hu S, Gao Y, Yan L. Pharmacokinetics of puerarin in the aqueous humor and vitreous of rabbit eye following systemic administration. Eye Sci 2006; 22: 275-279.
6. Wu C, Qi H, Chen W. Preparation and evaluation of a Carbopol/HPMC-based in situ gelling ophthalmic system for puerarin. J Pharm Soc Japan 2007; 127: 183-191.

7. Lane SS, Holland EJ. Loteprednol etabonate $0.5 \%$ versus prednisolone acetate $1.0 \%$ for the treatment of inflammation after cataract surgery. J Cataract Refract Surg 2013; 39: 168-173.

8. Wang C, Wang W, Jin X, Shen J, Hu W, Jiang T. Puerarin attenuates inflammation and oxidation in mice with collagen antibody-induced arthritis via TLR4/NF-kappaB signaling. Mol Med Rep 2016; 14: 1365-1370.

9. Singh AK, Jiang Y, Gupta S, Younus M, Ramzan M. Antiinflammatory potency of nano-formulated puerarin and curcumin in rats subjected to the lipopolysaccharideinduced inflammation. J Med Food 2013; 16: 899-911.

10. Zhang J, Li X, Gao Y. Effects of puerarin on the inflammatory role of burn-related procedural pain mediated by P2X (7) receptors. J Int Soc Burn Injuries 2013; 39: 610-618.

11. Luo ZK, Liu Y, Li HM. A clinical efficacy and safety study on coronary heart disease and angina treatment with Puerarin Injection. Zhonghua liuxingbingxue zazhi 2012; 33: 614-616.

12. Wu J, Zhang X, Zhang B. Efficacy and safety of puerarin injection in treatment of diabetic peripheral neuropathy: a systematic review and meta-analysis of randomized controlled trials. J Trad Chin Med 2014; 34: 401-410.

\section{*Correspondence to}

Xinying Liu

Department of 2nd Ophthalmology

The First Branch of Mudanjiang Medical University Affiliated Hongqi Hospital

Mudanjiang

Heilongjiang

P.R. China 